\title{
Dilated Vein of the Filum Terminale on MRI: A Marker for Deep Lumbar and Sacral Dural and Epidural Arteriovenous Fistulas
}

\author{
(D)W. Brinjikji, (DC.A. Hilditch, (D).M. Morris, (D)A.A. Dmytriw, (D) H. Cloft, (D) V. Mendes Pereira, (D) G. Lanzino, and (D). Krings
}

\begin{abstract}
BACKGROUND AND PURPOSE: Conventional MR imaging can provide important clues regarding the location of a spinal vascular malformation. We hypothesized that a dilated vein of the filum terminale, identified as a curvilinear flow void on T2WI, could be an imaging marker for a lower lumbar (L3-L5) or sacral fistula.
\end{abstract}

MATERIALS AND METHODS: We retrospectively identified all spinal dural and spinal epidural arteriovenous fistulas from 2 large tertiary referral centers from 2005 to 2018. All patients had a lumbar spinal MR imaging and a conventional spinal angiography. Images were reviewed by 2 neuroradiologists who categorized the level of the arterial feeder to the fistula and the presence or absence of a dilated vein of the filum terminale on $\mathrm{T} 2 \mathrm{WI}$ and $\mathrm{Tl}$ postcontrast images. We calculated the sensitivity, specificity, positive predictive value, and negative predictive value of the presence of a dilated filum terminale vein for a deep lumbar or sacral fistula.

RESULTS: One hundred sixty-two patients were included. An enlarged filum terminale vein was identified in 39 patients. Sensitivity, specificity, positive predictive value, and negative predictive value of the presence of a dilated filum terminale vein for a deep lumbar or sacral fistula were $86 \%, 98.3 \%, 94.9 \%$, and $95.1 \%$, respectively.

CONCLUSIONS: The presence of a dilated vein of the filum terminale can accurately localize a spinal dural arteriovenous fistula/spinal epidural arteriovenous fistula to the lower lumbar or sacral spine in patients being evaluated for such lesions. This finding can be used to facilitate both noninvasive and conventional spinal angiography.

ABBREVIATIONS: SDAVF = spinal dural arteriovenous fistula; SEDAVF = spinal epidural arteriovenous fistula; $V F T=$ vein of the filum terminale

S pinal dural arteriovenous fistulas (SDAVFs) and spinal epidural arteriovenous fistulas (SEDAVFs) are the 2 most common types of spinal vascular malformations. These lesions can result in considerable morbidity from congestive myelopathy secondary to chronic venous hypertension. ${ }^{1-6}$ Delay in the identification of spinal vascular malformations has been shown to result in high rates of irreversible morbidity due to the natural history of the disease, unnecessary surgeries including spinal cord biopsies and laminectomies, costly-yet-ineffective medical interventions including intravenous immunoglobulin therapy,

Received April 13, 2018; accepted after revision July 17

From the Departments of Radiology (W.B., J.M.M., H.C., G.L.) and Neurosurgery (W.B., G.L.), Mayo Clinic, Rochester, Minnesota; and Joint Department of Medical Imaging (W.B., C.A.H., A.A.D., V.M.P., T.K.), Toronto Western Hospital, Toronto, Ontario, Canada.

Timo Krings and Giuseppe Lanzino contributed equally as senior supervising authors for this work.

Please address correspondence to Waleed Brinjikji, MD, Mayo Clinic, 200 1st St SW, Rochester, MN 55905; e-mail: brinjikji.waleed@mayo.edu; @wbrinjikji

http://dx.doi.org/10.3174/ajnr.A5784 and substantial costs from unnecessary serologic and imaging investigations. $^{7}$

Once the imaging findings suggestive of a spinal vascular malformation are properly identified, further investigations are required to characterize the angioarchitecture and location of the dominant arterial feeders to the lesion. This is important for planning surgical and/or endovascular therapies. In many centers, a spinal CT angiogram or time-resolved large-FOV MR angiogram is obtained to get a sense of the location of the arterial feeders so that spinal angiography can be focused on a few levels. ${ }^{8-11}$ This is important because a complete spinal angiography can be timeconsuming and result in high radiation exposure for the patient and operator. A complete catheter spinal angiography also requires high volumes of iodinated contrast material, which can result in renal toxicity in some patients.

While noninvasive spinal angiography can be highly accurate in identifying the location of the fistula, it has limitations related to low spatial resolution (imaging typically requires a large FOV) and poor temporal resolution. While it was previously believed that conventional MR imaging findings of spi- 


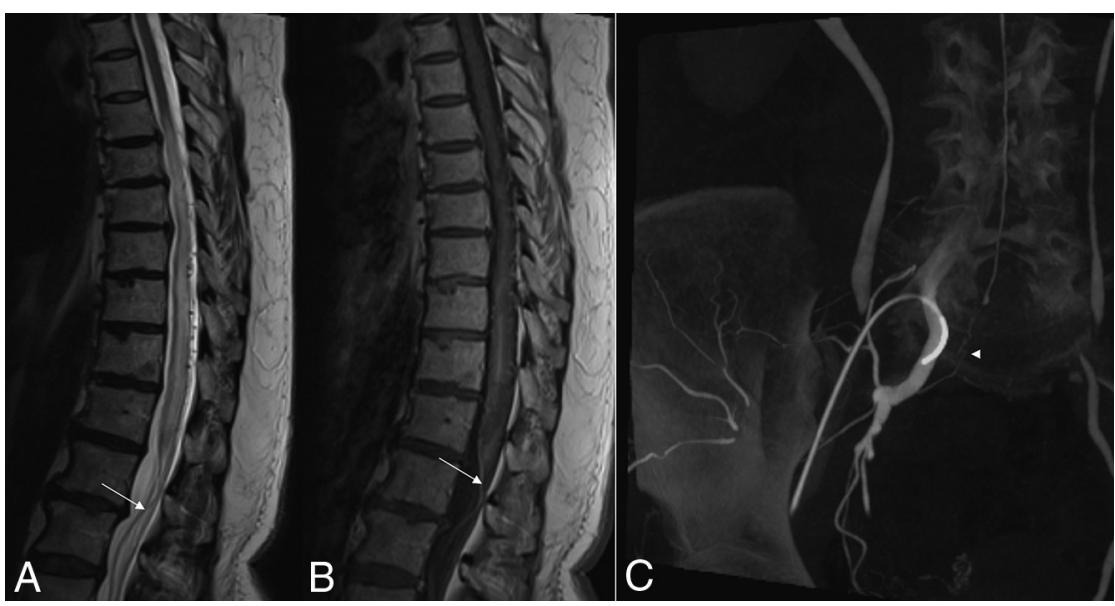

FIG 1. A 63-year-old woman with a sacral dural arteriovenous fistula at S1. A, Sagittal T2-weighted MR imaging shows a dilated vein of the filum terminale (white arrow). $B$, Contrast-enhanced $T$-weighted MR imaging again demonstrates the dilated vein of the filum terminale (white arrow). C, Rotational digital subtraction angiogram reformatted in the coronal plane following injection into the right internal iliac artery demonstrates a fistula at S1 (arrowhead), with a dilated vein of the filum terminale.
Outcomes and Statistical Analysis

The primary outcomes of this study were sensitivity, specificity, negative predictive value, positive predictive value, and accuracy of the presence of an enlarged VFT on conventional lumbar spine MR imaging in identifying lower lumbar and sacral fistulas. Lower lumbar and sacral fistulas were defined as fistulas located at the L3-S5 levels - the levels of the filum terminale. All statistical values are reported with their associated 95\% confidence intervals. A $\kappa$ statistic measuring interobserver agreement for the presence of a VFT was calculated as well. All statistical analyses were performed using JMP13.0 (SAS Institute, Cary, North Carolina). nal dural AVF such as the location of cord edema or the epicenter of the dilated perimedullary vessels cannot provide information about the location of the fistula, ${ }^{2}$ there have recently been a few case reports and smaller case series suggesting an association between a dilated vein of the filum terminale (VFT) and a deep lumbar or sacral AVF. ${ }^{12,13}$ We thus performed a retrospective review of SDAVFs and SEDAVFs from 2 tertiary referral centers to test the hypothesis that a dilated VFT, identified as a curvilinear midline flow void on T2WI or a dilated contrast-enhancing vessel below the conus on T1weighted postcontrast images, is an imaging marker for a lower lumbar (L3-L5) or sacral fistula.

\section{MATERIALS AND METHODS \\ Patient Selection}

Following institutional review board approval at 2 institutions, we retrospectively identified all patients with SDAVFs and SEDAVFs who underwent lumbar spinal MR imaging and conventional spinal angiography from 2005 to 2018. All patients had an angiographically confirmed SDAVF or SEDAVF. Children and patients who did not consent to the use of their charts for retrospective research were excluded. Patients with intramedullary, perimedullary, and metameric-type vascular malformations were excluded.

\section{Imaging Review}

Images were reviewed by 2 diagnostic and interventional neuroradiologists. MR images were reviewed for the presence of a dilated VFT, which was defined as a curvilinear flow void coursing along the filum terminale on sagittal T2-weighted images and/or a curvilinear enhancing VFT coursing along the filum on contrastenhanced T1-weighted images. The neuroradiologists were blinded to the level and type of fistula at the time of MR imaging interpretation. Following interpretation of the MR images, the 2 neuroradiologists reviewed the conventional spinal angiograms and identified the location of the arterial feeder to the spinal vascular malformation as well as the type of spinal vascular malformation (SEDAVF versus SDAVF).

\section{RESULTS}

\section{Patient Population and Baseline Characteristics}

One hundred sixty-two patients were included in this study. Mean patient age was $64.4 \pm 12.3$ years; $78.4 \%$ (127/162) of patients were men. There were 119 SDAVFs and 43 SEDAVFs. Forty-three fistulas $(26.5 \%)$ were deep lumbar or sacral, and 119 fistulas (73.4\%) were upper lumbar or thoracic. Of the 43 deep lumbar or sacral fistulas, 20 fistulas were sacral fistulas and 23 were deep lumbar fistulas. All patients had noncontrast lumbar spine MRIs with T2-weighted imaging in the sagittal plane, and 102 patients (63\%) had T1 contrast enhanced lumbar spine MR imaging in the sagittal plane.

\section{Dilated Vein of the Filum Terminale and Fistula Location}

Thirty-nine patients had a dilated VFT. Interobserver agreement for identifying the presence of a dilated VFT was excellent $(\kappa=$ 0.84; 95\% CI, 0.81-0.87). In all cases, a dilated VFT was present on both $\mathrm{T} 2$ and $\mathrm{T} 1$ contrast enhanced imaging.

The sensitivity of a dilated VFT in identifying a lower lumbar or sacral fistula was $86 \%$ (37/43; 95\% CI, 72.1\%-94.7\%). The specificity of a dilated VFT in identifying a lower lumbar or sacral fistula was $98.3 \%$ (117/119; 95\% CI, 94.1\%-99.8\%). The positive predictive value of a dilated VFT in identifying a lower lumbar or sacral fistula was 94.9\% (37/39; 95\% CI, $82.3 \%-98.7 \%)$. The negative predictive value of a dilated VFT in identifying a lower lumbar or sacral fistula was $95.1 \%$ (117/ 123; 95\% CI, 90.3\%-97.6\%). The accuracy of a dilated VFT in identifying a lower lumbar or sacral fistula was 95.1\% (154/ 162; 95\% CI, 90.5\%-97.8\%). The area under the curve of a dilated VFT in identifying a lower lumbar or sacral fistula was 0.921 . Ninety-five percent $(19 / 20)$ of sacral SEDAVFs and SDAVFs had a dilated VFT, and 78.3\% (18/23) of lower lumbar SEDAVFs and SDAVFs had a dilated VFT. Age, sex, and fistula type were not associated with the presence of a dilated VFT. Examples of dilated VFTs are provided in Figs 1-3. 


\section{DISCUSSION}

Our large retrospective study found that the presence of a dilated VFT on conventional T2WI is a reliable indicator for the presence of a lower lumbar or sacral spinal vascular malformation. Sensi-

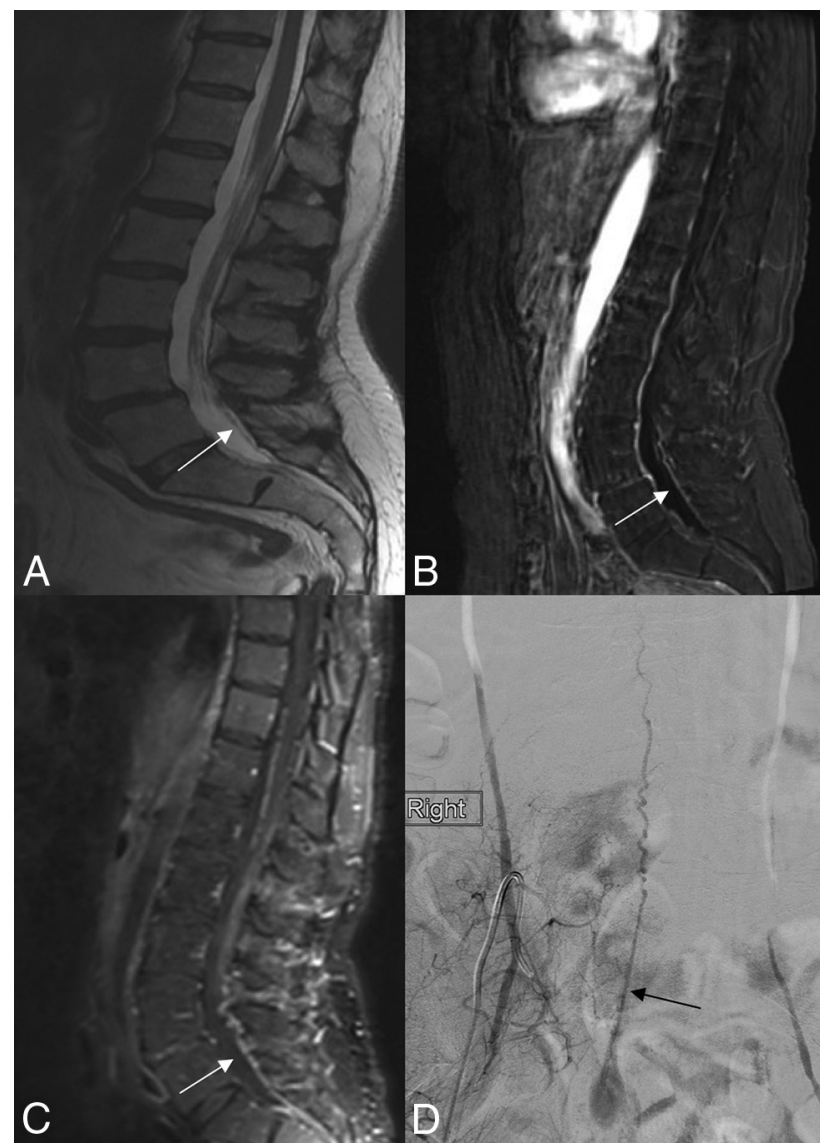

FIG 2. A 75-year-old man with a sacral dural arteriovenous fistula at S1. A, Sagittal T2-weighted MR imaging shows a dilated vein of the filum terminale (white arrow). $B$ and C, Contrast-enhanced MRIs demonstrate marked dilation of the vein of the filum terminale (white arrow). $D$, Conventional angiogram in the anteroposterior plane following injection into the right internal iliac artery demonstrates a fistula at S1 with a dilated vein of the filum terminale (black arrow).

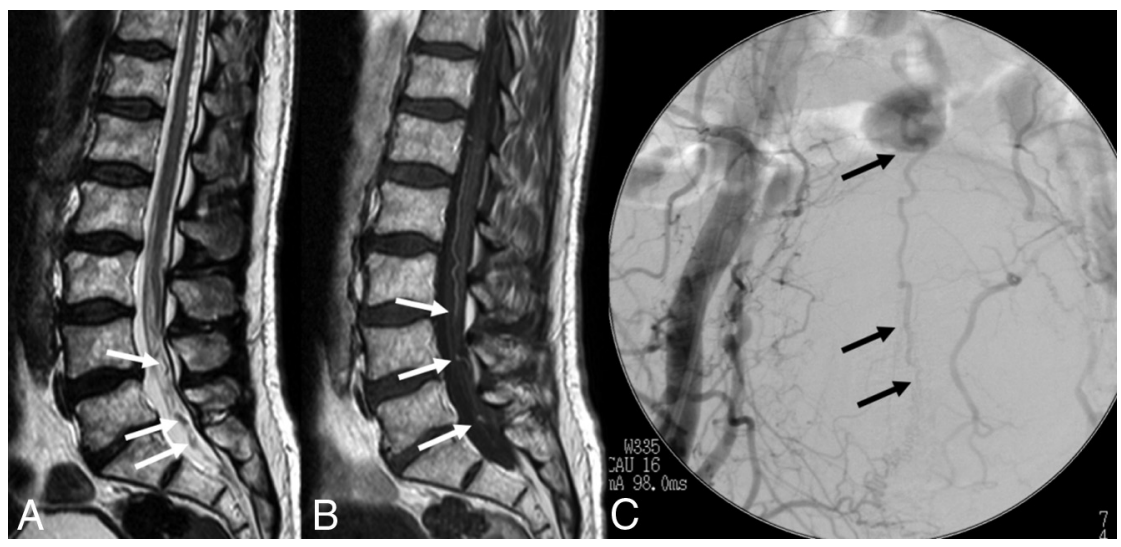

FIG 3. An 80-year-old man with a sacral dural arteriovenous fistula at S1. A, Sagittal T2-weighted MR imaging shows a dilated vein of the filum terminale (white arrows). $B$, Contrast-enhanced T1-weighted MR imaging again demonstrates the dilated vein of the filum terminale (white arrows). C, Conventional angiogram in the anteroposterior plane following injection into the right internal iliac artery demonstrates a fistula at S1 with a dilated vein of the filum terminale (black arrows). tivity, specificity, accuracy, negative predictive value, and positive predictive value were all high, and interobserver agreement was excellent. Ninety-five percent of sacral spinal vascular malformations were characterized by the presence of a dilated VFT, and $>80 \%$ of lower lumbar spinal vascular malformations had a dilated VFT. These findings are important because the identification of a dilated VFT can help in focusing noninvasive angiographic imaging and conventional spinal angiography on the lumbosacral levels, thus allowing more efficient identification of arterial feeders to these lesions.

The vascular supply to the filum terminale has been well-described in both radiographic and cadaveric studies. ${ }^{14,15}$ The filum terminale has a single artery arising from the termination of the anterior spinal artery, which travels along the anterior aspect of the filum into the sacral canal. The vein of the filum terminale, which is continuous with the anterior spinal vein, travels in front of the filum but behind the artery and is of uniform caliber along its course. There are no veins along the dorsal aspect of the filum. Because the VFT is the only intradural venous structure below the L2 vertebral body level, it is the only longitudinal collecting vein below this level. Thus, all dural and epidural AVFs with intradural venous drainage must drain through the VFT. ${ }^{14,15}$ The VFT can drain in 2 directions: 1 ) descending to the sacral venous plexus and hypogastric vein, and 2) ascending toward the anterior spinal vein along the spinal cord. It is thought that the ascending route is generally the preferred route. The propensity for the VFT to drain superiorly could explain the propensity for deep lumbar and sacral fistulas to result in a dilated VFT and the low rate of more superiorly located fistulas presenting with dilated VFTs. ${ }^{14,15} \mathrm{~A}$ small number of patients with deep lumbar and sacral fistulas did not have a dilated VFT on MR imaging. Angiographically, these lesions drained into the VFT; however, the VFT was likely not engorged enough to be easily seen on MR imaging.

Prior studies and case reports have shown high rates of VFT enlargement in the setting of deep lumbar and sacral fistulas. One of the first case reports demonstrating such an association was published by Chen and Hsu in 2002. ${ }^{12}$ The authors reported a sacral dural AVF, which presented with an enlarged VFT on sagittal T2-weighted MR imaging. The presence of such a vein prompted the investigators to pursue pelvic angiography before thoracolumbar angiography, resulting in prompt identification of the fistulous point. ${ }^{12}$ In a more recent series of sacral fistulas by Gioppo et al, ${ }^{16}$ the authors found that all 15 sacral fistulas had an enlarged VFT on MR imaging. Meanwhile, a recently published series of spinal vascular malformations at the L5 level or below found that $60 \%$ of fistulas had a dilated filum terminale vein, further reinforcing the association between a dilated VFT and the presence of a deep lumbar or sacral fistula. ${ }^{13}$ Our study differs from these prior studies because we are able to confirm that a dilated VFT identified using conventional 
MR imaging techniques is a sensitive and specific sign for localization of SDAVF/SEDAVFs to the lower lumbar or sacral spine in patients being evaluated for such lesions.

Numerous studies have demonstrated the utility of various MR and CT angiographic techniques in identifying the feeding artery and angioarchitecture of spinal vascular malformations. ${ }^{3,17-19}$ These techniques have proved instrumental in focusing spinal angiography to make conventional spinal angiography more efficient and reduce operative time, radiation exposure, and contrast dose. However, these techniques have their limitations. For gadolinium bolus spinal angiography, some centers lack the capability and scanner time to image the entire spine in patients with suspected spinal vascular malformations. Thus, such centers will often perform a gadolinium bolus MRA focused on the thoracic and upper lumbar spine and will miss deep lumbar and sacral fistulas on initial evaluation. ${ }^{20}$ This can be mitigated by performing 2 gadolinium-bolus MRAs; however, it is preferable to avoid this situation. In the setting of noninvasive 4D MR imaging, there is often a trade-off among spatial, temporal, and contrast resolution. ${ }^{20}$ Thus, the ability to narrow the FOV to a smaller region in the setting of a dilated VFT could allow improved noninvasive characterization of spinal vascular malformations. ${ }^{20}$ Our study suggests that when a dilated VFT is identified, radiologists should focus their noninvasive angiographic investigations in the lumbosacral region, including branches of the iliac arteries. We have found that lack of adequate angiographic investigation of the iliac branches is one of the most common reasons for missing spinal vascular malformations on false-negative spinal angiographic studies. ${ }^{7}$

\section{Limitations}

Our study has limitations. First, given its retrospective nature, there is a propensity for selection bias. We included only patients with SDAVFs and SEDAVFs; thus, our results do not apply to fistulas of the filum terminale, pial AVFs, and nidus-type AVMs. A fistula of the filum terminale will, by definition, have a dilated VFT. ${ }^{21}$ Thus, there is the potential for an overreliance on this sign to cause clinicians to miss such a lesion. However, these lesions are also characterized by a markedly enlarged anterior spinal artery. The patients included in this study were imaged during a 13-year period using various MR imaging scanners, MR imaging protocols, and various field strengths (1.5T versus $3 \mathrm{~T}$ ). This feature can limit the generalizability of our results.

\section{CONCLUSIONS}

Our retrospective study including $>160$ patients with SDAVFs and SEDAVFs and lumbar spine imaging found that the presence of a dilated VFT is sensitive and specific for localization of SEDAVF/SDAVFs to the lower lumbar or sacral spine in patients being evaluated for such lesions. Our findings suggest that the presence of an enlarged VFT should prompt the neuroangiographer to initially catheterize the lower lumbar and internal iliac vessels when attempting to identify the location and arterial feeders of a SEDAVF/SDAVF. Our results are also valuable in planning noninvasive spinal angiography by allowing imagers to narrow their FOVs or center on the lumbosacral region.

\section{REFERENCES}

1. Krings T, Geibprasert S. Spinal dural arteriovenous fistulas. AJNR Am J Neuroradiol 2009;30:639-48 CrossRef Medline

2. Krings T, Mull M, Gilsbach JM, et al. Spinal vascular malformations. Eur Radiol 2005;15:267-78 CrossRef Medline

3. Morris JM. Imaging of dural arteriovenous fistula. Radiol Clin North Am 2012;50:823-39 CrossRef Medline

4. Morris JM, Kaufmann TJ, Campeau NG, et al. Volumetric myelographic magnetic resonance imaging to localize difficult-to-find spinal dural arteriovenous fistulas. J Neurosurg Spine 2011;14:398404 CrossRef Medline

5. Brinjikji W, Yin R, Nasr DM, et al. Spinal epidural arteriovenous fistulas. J Neurointerv Surg 2016 Jan 20. [Epub ahead of print] CrossRef Medline

6. Nasr DM, Brinjikji W, Clarke MJ, et al. Clinical presentation and treatment outcomes of spinal epidural arteriovenous fistulas. J Neurosurg Spine 2017;26:613-20 CrossRef Medline

7. Brinjikji W, Nasr DM, Morris JM, et al. Clinical outcomes of patients with delayed diagnosis of spinal dural arteriovenous fistulas. $A J N R$ Am J Neuroradiol 2016;37:380-86 CrossRef Medline

8. Condette-Auliac S, Boulin A, Roccatagliata L, et al. MRI and MRA of spinal cord arteriovenous shunts. J Magn Reson Imaging 2014;40: 1253-66 CrossRef Medline

9. Lindenholz A, TerBrugge KG, van Dijk JM, et al. The accuracy and utility of contrast-enhanced MR angiography for localization of spinal dural arteriovenous fistulas: the Toronto experience. Eur Radiol 2014;24:2885-94 CrossRef Medline

10. Oda S, Utsunomiya D, Hirai T, et al. Comparison of dynamic contrast-enhanced 3T MR and 64-row multidetector CT angiography for the localization of spinal dural arteriovenous fistulas. AJNR Am J Neuroradiol 2014;35:407-12 CrossRef Medline

11. Amarouche M, Hart JL, Siddiqui A, et al. Time-resolved contrastenhanced MR angiography of spinal vascular malformations. AJNR Am J Neuroradiol 2015;36:417-22 CrossRef Medline

12. Chen CJ, Hsu HL. Engorged and tortuous intradural filum terminale vein as a sign of a sacral dural arteriovenous malformation. Eur J Radiol 2002;44:152-55 CrossRef Medline

13. Jablawi F, Nikoubashman O, Schubert GA, et al. Clinical and radiologic characteristics of deep lumbosacral dural arteriovenous fistulas. AJNR Am J Neuroradiol 2018;39:392-98 CrossRef Medline

14. Djindjian M, Ribeiro A, Ortega E, et al. The normal vascularization of the intradural filum terminale in man. Surg Radiol Anat 1988;10: 201-09 CrossRef Medline

15. Namba K. Vascular anatomy of the cauda equina and its implication on the vascular lesions in the caudal spinal structure. Neurol Med Chir (Tokyo) 2016;56:310-16 CrossRef Medline

16. Gioppo A, Faragò G, Giannitto C, et al. Sacral dural arteriovenous fistulas: a diagnostic and therapeutic challenge-single-centre experience of 13 cases and review of the literature. J Neurointerv Surg 2018;10:415-21 CrossRef Medline

17. Lai PH, Pan HB, Yang CF, et al. Multi-detector row computed tomography angiography in diagnosing spinal dural arteriovenous fistula: initial experience. Stroke 2005;36:1562-64 CrossRef Medline

18. Lai PH, Weng MJ, Lee KW, et al. Multidetector CT angiography in diagnosing type I and type IVA spinal vascular malformations. AJNR Am J Neuroradiol 2006;27:813-17 Medline

19. Mathur S, Symons SP, Huynh TJ, et al. First-pass contrast-enhanced MRA for pretherapeutic diagnosis of spinal epidural arteriovenous fistulas with intradural venous reflux. AJNR Am J Neuroradiol 2017; 38:195-99 CrossRef Medline

20. Mathur S, Bharatha A, Huynh TJ, et al. Comparison of time-resolved and first-pass contrast-enhanced MR angiography in pretherapeutic evaluation of spinal dural arteriovenous fistulas. AJNR Am J Neuroradiol 2017;38:206-12 CrossRef Medline

21. Giordan E, Brinjikji W, Ciceri E, et al. Arteriovenous fistulae of the filum terminale. J Neurointerv Surg 2018;10:191-97 CrossRef Medline 